\title{
Formação de Sequências: Aspectos Conceituais e Metodológicos
}

\author{
Grauben José Alves de Assis \\ Marcelo Quintino Galvão Baptista \\ Ana Letícia Moraes Nunes \\ Universidade Federal do Pará
}

\begin{abstract}
RESUMO
A noção de controle de estímulos pode ser aplicada à análise de comportamentos novos, derivados de contingências que estabelecem a produção de sequências, nas quais a ordem tem uma importante função. O presente estudo teve como objetivo apontar algumas descrições comportamentais sobre o responder sequencial, sugeridas por alguns autores. Apresenta alguns aspectos conceituais referentes à relação entre topografia de controle de estímulos e aprendizagem relacional; tipos de sequências; e a definição de sequências baseada em propriedades matemáticas e outras possíveis fontes de controle. Os estudos relatados apresentaram em comum um treino por encadeamento ou sobreposição de estímulos que constituíam a linha de base para exposição aos testes de transitividade, conectividade e formação de classes ordinais. Conclui-se que no responder sequencial, a variável ordem se define a partir de dimensões ou características de estímulos com as quais tem relação, dentre as quais o tamanho da sequência e a temporalidade e remete à relação entre eventos antecedentes e consequentes.
\end{abstract}

Palavras-chave: produção de sequencias; encadeamento; humanos.

\begin{abstract}
Sequence Formation: Conceptual and Methodological Aspects

The idea of stimulus control can be applied to the analysis of new behavior, which derives from contingencies that establishes the production of sequences, in which order has an important function. The present study had as its objective to point out some behavioral descriptions about sequential responding, as suggested by previous authors. It presents some conceptual aspects about the relation between stimulus control topography and relational learning; the kinds of sequences; and the definition of sequences based on mathematical properties and other possible sources of control. The studies we reviewed have in common the chaining training or stimulus overlapping, which constitutes the baseline for the exposure to the transitivity, connectivity and ordinal classes formation tests. We conclude that in sequential responding, order as a variable is defined by the dimensions or characteristics of stimuli to which it is related - among them, the length of the sequence and temporality. Furthermore, it refers to the relation between antecedent and consequent events.
\end{abstract}

Keywords: sequence production; chaining; humans.

Defende-se neste artigo que a noção de controle de estímulos pode ser aplicada à análise de comportamentos novos, derivados de contingências que estabelecem a produção de sequências, nas quais a ordem tem uma importante função (cf. Green, Stomer \& Mackay, 1993). Vários estudos sobre a produção de sequências têm buscado a compreensão de como repertórios sob controle da ordem são estabelecidos e mantidos. Esses estudos têm considerado diferentes processos comportamentais envolvidos no responder sequencial: a) a inferência transitiva (Green, Stromer \& Mackay, 1993; Holcomb, Stromer \& Mackay 1997;
Lopes Jr. \& Agostini, 2004; Stromer \& Mackay, 1990; Stromer \& Mackay, 1993); b) a formação de classes sequenciais em contingência tríplice (Assis \& Sampaio, 2003; Galy, Camps \& Melan, 2003; Lima \& Assis, 2003; Sampaio \& Assis, 2005; Souza, R \& Assis, 2005; Stromer \& Mackay, 1993; Stromer, Mackay, Cohen \& Stoddard, 1992); c) o controle condicional (Assis \& Costa, 2004; Lazar \& Kotlarchyk, 1986; Stromer \& Mackay, 1992a, Stromer \& Mackay, 1992b; Wulfert \& Hayes, 1988); d) a transferência de desempenhos de matching to sample para classes sequenciais ou vice-versa (Green, Sigurdardottir, \& 
Saunders, 1991; Lazar, 1977; Mackay, Kotlarchyk \& Stromer, 1997; Maydack, Stromer, Mackay \& Stoddard, 1995; Nunes \& Assis, 2006; Ribeiro, Assis \& Enumo, 2005; Sigudardotir, Green \& Saunders, 1991).

O presente estudo teve como objetivo apontar algumas descrições comportamentais sobre o responder sequencial, sugeridas por alguns autores - por exemplo, a relação entre topografia de controle de estímulos e aprendizagem relacional; os tipos de sequências e a definição de sequências baseada em propriedades matemáticas.

\section{TOPOGRAFIA DE CONTROLE DE ESTÍMULOS E APRENDIZAGEM RELACIONAL}

Explicações para o controle de desempenhos sequenciais têm sido buscadas na análise da relação entre conceitos como topografia de controle de estímulos e aprendizagem relacional. Dentre os eventos ambientais, a topografia de controle de estímulos refere-se às características, relações estruturais e propriedades controladoras dos estímulos (McIlvane \& Dube, 1992; 2003). Uma mesma resposta pode ocorrer em diferentes topografias de controle de estímulos (TCEs), ou seja, em função de várias combinações de propriedades (forma, cor, tamanho, posição, textura, numerosidade etc.) da situação. Por exemplo, em uma tarefa de discriminação simples com dupla escolha envolvendo formas como estímulos $\mathrm{S}^{+}$e $\mathrm{S}^{-}$, as quais são apresentadas em igual frequência nas posições esquerda e direita de um painel, duas possíveis TCEs poderiam controlar o responder: 1) a forma física do estímulo definido como "correto" e 2) a posição do estímulo, ou o fato de este aparecer em um dos lados (por exemplo, esquerdo) do painel (posição), a despeito da forma deste. Escolhas com base na forma física do estímulo, definida como correta, são sempre reforçadas. É possível também que as escolhas realizadas com base na posição do estímulo sejam reforçadas em uma proporção de tentativas. Isto é, se o participante responder exclusivamente ao estímulo posicionado à esquerda, metade dessas respostas seria seguida por reforço. Essa porcentagem de reforçamento poderá ser suficiente para manter o controle de estímulos pela posição, em vez da forma definida como $\mathrm{S}^{+}$(para detalhes do procedimento, ver Serna, 2004).

\section{TIPOS DE SEQUÊNCIAS}

Segundo Kelleher (1966), as sequências comportamentais têm sido estudadas como cadeias de respostas, área de estudos tradicionalmente denominada de encadeamento. O conceito de encadeamento foi, inicialmente, incorporado da fisiologia, na abordagem de comportamentos reflexos (Sherrington, 1906, citado por Kelleher, 1966). Depois, foi estendido a sequências comportamentais aprendidas, como reflexos condicionados (Smith \& Guthrie, 1921, citado por Kelleher, 1966). Finalmente, Skinner (1938) introduziu o conceito de cadeia operante.

No procedimento de ensino por encadeamento, cada estímulo é introduzido gradativamente na sequência. Em uma cadeia de respostas “...uma sucessão de operantes diferentes, cada um definido pela consequência reforçadora de produzir uma oportunidade de emitir o próximo, até que a sequência seja terminada por um reforçador" (Catania, 1999, p. 142). Ou seja, na cadeia, cada estímulo "tem a dupla função, reforçar a resposta que vem antes e ser um $\mathrm{S}^{\mathrm{D}}$ para a que vem depois" (Millenson, 1975, p. 245).

Outro procedimento que tem sido usado para ensinar uma sequência de respostas é o de sobreposição de estímulos. Nesse procedimento, dois estímulos são apresentados simultaneamente (por exemplo, A1 $\rightarrow$ A2). O participante precisa responder ao primeiro e depois ao segundo estímulo para produzir o reforço. Em seguida, um terceiro estímulo é introduzido e o primeiro removido da sequência (por exemplo, A2 $\rightarrow$ A3) e assim sucessivamente até se completar a sequência.

Spradlin (1999) descreveu um exemplo específico de sequência comportamental: as rotinas. O autor refere-se à rotina como uma sequência repetitiva de estímulos e respostas topograficamente diferentes (similar a uma cadeia) que ocorrem em uma situação típica. Alguns comportamentos seguem uma ordem fixa, em função da natureza do ambiente físico. Por exemplo, ao dirigir um carro, é preciso colocar a chave no contato, depois virá-la à direita para acionar o motor, em seguida, trocar de marcha, e assim por diante. Rotinas desse tipo produzem, provavelmente, um menor custo de resposta. Algumas dessas rotinas podem ter uma variação na sua ordem, tornando-se mais flexíveis do que outras, como, por exemplo, as rotinas matinais indicando que se pode levantar e ir ao banheiro ou à cozinha preparar o café. As rotinas variáveis, ao contrário das fixas, geram, provavelmente, um maior custo de resposta.

Spradlin (1999) apresentou dois termos usados em menção à extensão e à complexidade das sequências comportamentais: sequência molar e sequência molecular. "O que é um componente em um nível de análise pode ser uma rotina em outro nível de análise" 
(Spradlin, 1999, p. 226). Segundo Skinner (2000/ 1953), uma cadeia bem integrada pode ser tratada como uma unidade de comportamento operante. Segundo o autor, "muitas vezes lidamos com uma cadeia como uma única resposta" (p. 245). De fato, pode-se abordar a rotina como uma unidade ou considerar seus componentes. Uma análise molar poderia enfatizar rotina como um todo, em detrimento da ênfase aos comportamentos que a constituem. Por exemplo, para um garçom experiente, seria suficiente uma solicitação genérica no sentido de preparar um jantar. Ao contrário, um garçom pouco experimente exigiria uma solicitação bem detalhada para o preparo do jantar. No primeiro caso, a solicitação corresponde a uma sequência verbal molar; no segundo caso, a solicitação corresponde a uma sequência verbal molecular. De acordo com Spradlin (1999), o nível particular de uma análise dependerá de considerações práticas.

\section{DEFINIÇÃO DE SEQUÊNCIA COM BASE EM PROPRIEDADES MATEMÁTICAS}

$O$ responder sequencial foi estudado em termos de cadeias de estímulos e respostas (Skinner, 1938), mas recentemente foram levantadas questões sobre a adequação de tal análise (Green e cols., 1993). Algumas pesquisas demonstraram que o treino por encadeamento ou por sobreposição produz a formação de classes de estímulos baseadas na posição ordinal dentro da sequência ensinada (Lazar, 1977; Sigurdardottir, Green \& Saunders, 1990; Stromer \& Mackay, 1993; Stromer, Mackay, Cohen \& Stoddard, 1992; Wulfert \& Hayes, 1988). Esses estudos levaram Green e cols. (1993) a descreverem uma proposta alternativa de análise das relações entre estímulos em uma sequência de estímulos e entre sequências ensinadas separadamente. Segundo Green e cols. (1993), relações entre os componentes das sequências de estímulos podem ser consideradas relações ordinais, se for possível identificar, entre os mesmos, cada uma das propriedades de irreflexividade, assimetria, transitividade e conectividade (Stevens, 1951), por meio de testes comportamentais.

A definição matemática de uma relação ordinal foi tomada como guia para uma análise descritiva e organizada de relações estímulo-estímulo produzidas por um treino sequencial envolvendo discriminação simples. Para isso, Green e cols. (1993) propuseram códigos alfanuméricos para representar estímulos e o símbolo " $\rightarrow$ " para indicar uma relação de ordem. Por exemplo, a sequência "A1 $\rightarrow \mathrm{A} 2 \rightarrow \mathrm{A} 3 \rightarrow \mathrm{A} 4 \rightarrow \mathrm{A} 5$ " representa cinco estímulos diferentes aos quais se responde numa dada ordem.

Irreflexividade pressupõe que uma relação ordinal não é reflexiva. Assim, não é verdade, por exemplo, que $\mathrm{A} 1 \rightarrow \mathrm{A} 1$. A propriedade da assimetria pressupõe que uma relação ordinal deve ser unidirecional; assim, se $\mathrm{A} 2 \rightarrow \mathrm{A} 3$ é verdadeira, então $\mathrm{A} 3 \rightarrow \mathrm{A} 2$ não pode ser considerada uma relação correta. Se, por exemplo, $\mathrm{A} 2 \rightarrow \mathrm{A} 3$ e $\mathrm{A} 3 \rightarrow \mathrm{A} 4$, então $\mathrm{A} 2 \rightarrow \mathrm{A} 4$ é correta, na ausência do A3. Essa relação, A2 $\rightarrow \mathrm{A} 4$, expressa a propriedade da transitividade. Note-se que a transitividade envolve pares não adjacentes dentro de séries. Finalmente, se $\mathrm{A} 1 \rightarrow \mathrm{A} 2 \rightarrow \mathrm{A} 3$, então $\mathrm{A} 1 \rightarrow \mathrm{A} 2, \mathrm{~A} 1 \rightarrow \mathrm{A} 3 \mathrm{e}$ $\mathrm{A} 2 \rightarrow \mathrm{A} 3$, ou seja, é verdadeira a relação denominada de conectividade entre todos os pares possíveis.

O teste de todas essas propriedades tem sido conduzido sem reforçamento diferencial e baseado nas relações ensinadas, consistindo na verificação da recombinação de estímulos em uma contingência tríplice. O modelo comportamental para sequências é basicamente uma expansão da proposta de Sidman e Tailby (1982) sobre as relações entre estímulos em classes equivalentes. Tal modelo fornece uma estratégia para o exame da interação entre equivalência e relações ordinais quando leva à ampliação de repertórios comportamentais, conforme pode ser observado no relato dos estudos aqui apresentados.

\section{FONTES DE CONTROLE A SEREM IDENTIFICADAS}

Segundo Catania (1999), uma preocupação relevante diz respeito à determinação das fontes de controle de alguns desempenhos sequenciais que parecem não depender de sequências de estímulo-resposta. Por exemplo, é difícil identificar o que controla a sucessão rápida de movimentos de um músico em determinados trechos de uma composição. A análise de que cada movimento envolvido no tocar um instrumento musical serve como um estímulo discriminativo para o próximo, como se postula para a descrição da aprendizagem por encadeamento, pode não ser útil para explicar esse responder.

Mais recentemente, um termo, fluência, foi sugerido na literatura como importante para a definição de desempenhos (de operante livre) precisos envolvendo a possibilidade de sequência (cf. Binder, 1996; Johnson \& Laying; 1996). Fluência comportamental é a combinação de precisão e velocidade no responder que caracterizam desempenhos competentes em ambientes naturais. Como hipótese, sugere-se que o controle sobre desempenhos fluentes decorre, predominante- 
mente, de estímulos proprioceptivos. É possível que isso ajude na explicação de comportamentos como aqueles descritos por Catania (1999) e, em particular, clarifique a compreensão dos movimentos envolvidos no tocar.

Outra base ou fonte de controle do responder sequencial, segundo Carmo (2002), abrange, em parte, a proposta de Green e cols. (1993), mas exclui as propriedades de irreflexividade, assimetria, transitividade e conectividade, sugeridas e que se fundamentam na matemática. Ou seja, interpreta-se que, na análise de Carmo (2002), a formação de sequência é, pelo menos implicitamente, um desempenho que expressa o controle pela ordem, no sentido de que, no responder sequencial, a primeira resposta de escolha é determinada por um ou outro tipo de dimensão de estímulo; portanto, a ordem em que as escolhas ocorrem caracteriza a temporalidade, ou seja, o sequenciar é um desempenho em que há sucessão de respostas. Ao admitir a existência da ordem no controle da formação de sequência, implicitamente, a análise de Carmo é similar à concepção de Green e cols. (1993). Porém, em termos explícitos, essa análise privilegia o controle por dimensões ou características de estímulos tais como tamanho, forma, substância, numerosidade e outras, o que remete à noção de topografia de controle de estímulos apresentada por Mcllvane e Dube (1992), ratificada posteriormente (Dube \& McIlvane, 1996; McIlvane \& Dube, 2003). Um exemplo elucida a análise de Carmo (2002), evidenciando, explicitamente, o controle da formação de sequência por dimensões de estímulos: uma criança, ao dispor brinquedos em sequência, pode ficar sob controle do tipo de material de que são confeccionados: "primeiro, os brinquedos de plástico, em seguida os de madeira" (p. 187; grifos do autor). Carmo sustenta que esse exemplo não ilustra o ordenar, mas é sim "um caso de sequenciação", como sendo "ato ou efeito de sequenciar" (p. 187).
A seguir serão descritos vários estudos que compõem uma linha de pesquisa em andamento.

\section{CARACTERIZAÇÃO GERAL DOS ESTUDOS}

$\mathrm{Na}$ Tabela 1 apresentada a seguir, identifica-se cada estudo, caracterizando-o pelo procedimento utilizado, tipo de tentativa de treino e de testes, e os resultados dos testes de conectividade. Todos os procedimentos de treino e testes foram informatizados. Os resultados de cada um dos sete estudos foram analisados como estudos que seguiram a proposta de Green e cols. (1993). Os testes comportamentais que avaliaram a emergência de novas sequências, a partir daquela estabelecida na linha de base por meio de encadeamento, faziam parte de todos esses estudos, à exceção de um, em diferentes arranjos experimentais, tendo em conta as propriedades matemáticas definidoras de sequência, ou seja, a irreflexividade, assimetria, transitividade e conectividade. O conjunto de estudos envolveu diferentes participantes: universitários (Estudo 1 - Lima \& Assis, 2003); alunos com atraso no desenvolvimento cognitivo e diferentes idades cronológica e mental (Estudos 2, 4, 6 e 7 - Assis \& Sampaio, 2003; Nunes \& Assis, 2006; Sampaio \& Assis, 2005; Souza, J \& Assis, 2005); alunos com deficiência auditiva (Estudo 5 - Souza \& Assis, 2005) e crianças, entre 4 e 9 anos de idade, de ambos os sexos (Estudo 3 - Assis \& Costa, 2004). Diferentes variáveis de procedimentos estavam envolvidas nos estudos: pareamento consistente (Estudo 1); pareamento com o modelo e encadeamento (Estudo 7); encadeamento (Estudo 2, 3, 4, 5, 6 e 7); encadeamento e sobreposição de estímulos (Estudo 2 e 4); tipos de estímulos experimentais: numerais (Estudos 1 e 7); nomes de numerais (Estudo 5 e 7); sinais de numerais em LIBRAS (Estudo 5); cores (Estudos 3 e 5); figuras usuais ou facilmente relacionáveis com o cotidiano (Estudo 6); figuras não usuais, ou seja, de difícil relação com o cotidiano, como formas abstratas (Estudos 1, 3, 4, 5 e 7). 
TABELA 1

Caracterização dos Estudos 1, 2, 3, 4, 5, 6 e 7 (Lima \& Assis, 2003; Assis \& Sampaio, 2003; Assis \& Costa, 2004; Souza, J. \& Assis, 2005; Souza, R. \& Assis, 2005; Sampaio \& Assis, 2005; Nunes \& Assis, 2006), Procedimento Utilizado, Tipo de Tentativa de Treino, Tipo de Tentativa de Teste de Conectividade e Resultados no Teste

\begin{tabular}{|c|c|c|c|c|}
\hline Estudos & Procedimento & $\begin{array}{c}\text { Treino } \\
\text { Tipo de Tentativa }\end{array}$ & $\begin{array}{c}\text { Testes } \\
\text { Tipo de Tentativa } \\
\text { Conectividade } \\
\end{array}$ & $\begin{array}{l}\text { Resultados } \\
\text { do Teste }\end{array}$ \\
\hline \multirow{4}{*}{$\begin{array}{l}\text { 1. Lima \& Assis } \\
\text { (2003) }\end{array}$} & \multirow{4}{*}{$\begin{array}{l}\text { - Treino AB, AC, AD. } \\
\text { - Testes de Simetria BA, CA, DA. } \\
\text { - Testes de Produção de Sequências, } \\
\text { Transitividade e Conectividade. }\end{array}$} & AB misto & $\mathrm{B} 1 \rightarrow \mathrm{C} 2 \rightarrow \mathrm{C} 3 \rightarrow \mathrm{B} 4 \rightarrow \mathrm{C} 5$ & 1 \\
\hline & & $\mathrm{AC}$ misto & $\mathrm{C} 1 \rightarrow \mathrm{B} 2 \rightarrow \mathrm{C} 3 \rightarrow \mathrm{B} 4 \rightarrow \mathrm{B} 5$ & 2 \\
\hline & & AD misto & $\mathrm{D} 1 \rightarrow \mathrm{B} 2 \rightarrow \mathrm{D} 3 \rightarrow \mathrm{D} 4 \rightarrow \mathrm{B} 5$ & 2 \\
\hline & & & $\mathrm{C} 1 \rightarrow \mathrm{D} 2 \rightarrow \mathrm{C} 3 \rightarrow \mathrm{B} 4 \rightarrow \mathrm{D} 5$ & 2 \\
\hline \multirow{9}{*}{\multicolumn{2}{|c|}{$\begin{array}{ll}\text { 2. Assis \& Sampaio - Grupo 1: Treino por sobreposição e } \\
\text { (2003) } & \text { encadeamento de duas sequências. } \\
& \text { - Teste de Transitividade e Conectividade. }\end{array}$}} & Sobreposição & $\mathrm{W} 1 \rightarrow \mathrm{X} 2 \rightarrow \mathrm{W} 3 \rightarrow \mathrm{X} 4 \rightarrow \mathrm{W} 5$ & 2 \\
\hline & & $\mathrm{X} 1 \rightarrow \mathrm{X} 2$ & $\mathrm{X} 1 \rightarrow \mathrm{W} 2 \rightarrow \mathrm{X} 3 \rightarrow \mathrm{W} 4 \rightarrow \mathrm{X} 5$ & 2 \\
\hline & & $\mathrm{X} 2 \rightarrow \mathrm{X} 3$ & & \\
\hline & & $\mathrm{X} 3 \rightarrow \mathrm{X} 4$ & & \\
\hline & & $X 4 \rightarrow X 5$ & & \\
\hline & & $\mathrm{W} 1 \rightarrow \mathrm{W} 2$ & & \\
\hline & & $\mathrm{W} 2 \rightarrow \mathrm{W} 3$ & & \\
\hline & & W3 $\rightarrow$ W4 & & \\
\hline & & $\mathrm{W} 4 \rightarrow \mathrm{W} 5$ & & \\
\hline & \multirow{3}{*}{$\begin{array}{l}\text { - Grupo 2: Treino por encadeamento e } \\
\text { sobreposição de duas sequências. } \\
\text { - Teste de Transitividade e Conectividade. }\end{array}$} & Encadeamento & & 1 \\
\hline & & $\mathrm{X} 1 \rightarrow \mathrm{X} 2 \rightarrow \mathrm{X} 3 \rightarrow \mathrm{X} 4 \rightarrow \mathrm{X} 5$ & $\mathrm{~W} 1 \rightarrow \mathrm{X} 2 \rightarrow \mathrm{W} 3 \rightarrow \mathrm{X} 4 \rightarrow \mathrm{W} 5$ & 1 \\
\hline & & $\mathrm{W} 1 \rightarrow \mathrm{W} 2 \rightarrow \mathrm{W} 3 \rightarrow \mathrm{W} 4 \rightarrow \mathrm{W} 5$ & $\mathrm{X} 1 \rightarrow \mathrm{W} 2 \rightarrow \mathrm{X} 3 \rightarrow \mathrm{W} 4 \rightarrow \mathrm{X} 5$ & \\
\hline \multirow[t]{12}{*}{$\begin{array}{l}\text { 3. Assis \& Costa } \\
(2004)\end{array}$} & $\begin{array}{l}\text { - Experimento 1: Treino por Encadeamento } \\
\text { de quatro sequências. }\end{array}$ & $\begin{array}{c}\text { Experimento } 1 \\
A 1 \rightarrow A 2 \rightarrow A 3 \rightarrow A 4 \rightarrow A 5\end{array}$ & $\mathrm{~A} 1 \rightarrow \mathrm{B} 2 \rightarrow \mathrm{A} 3 \rightarrow \mathrm{B} 4 \rightarrow \mathrm{A} 5$ & 1 \\
\hline & \multirow{3}{*}{$\begin{array}{l}\text { - Testes de Produção de Sequências, } \\
\text { Transitividade e Conectividade. }\end{array}$} & $\mathrm{B} 1 \rightarrow \mathrm{B} 2 \rightarrow \mathrm{B} 3 \rightarrow \mathrm{B} 4 \rightarrow \mathrm{B} 5$ & $\mathrm{~B} 1 \rightarrow \mathrm{A} 2 \rightarrow \mathrm{B} 3 \rightarrow \mathrm{A} 4 \rightarrow \mathrm{B} 5$ & 1 \\
\hline & & $\mathrm{C} 1 \rightarrow \mathrm{C} 2 \rightarrow \mathrm{C} 3 \rightarrow \mathrm{C} 4 \rightarrow \mathrm{C} 5$ & $\mathrm{C} 1 \rightarrow \mathrm{D} 2 \rightarrow \mathrm{C} 3 \rightarrow \mathrm{D} 4 \rightarrow \mathrm{C} 5$ & 2 \\
\hline & & $\mathrm{D} 1 \rightarrow \mathrm{D} 2 \rightarrow \mathrm{D} 3 \rightarrow \mathrm{D} 4 \rightarrow \mathrm{D} 5$ & $\mathrm{D} 1 \rightarrow \mathrm{C} 2 \rightarrow \mathrm{D} 3 \rightarrow \mathrm{C} 4 \rightarrow \mathrm{D} 5$ & 2 \\
\hline & $\begin{array}{l}\text { - Experimento 2: Treino por Encadeamento } \\
\text { de duas sequências, com estímulos } \\
\text { condicionais (cores). }\end{array}$ & & & \\
\hline & \multirow[t]{7}{*}{$\begin{array}{l}\text { - Testes de Produção de Sequências, } \\
\text { Transitividade e Conectividade. }\end{array}$} & Experimento 2 & & 1 \\
\hline & & Verde & Verde $-\mathrm{A} 1 \rightarrow \mathrm{B} 2 \rightarrow \mathrm{A} 3 \rightarrow \mathrm{B} 4 \rightarrow \mathrm{A} 5$ & \\
\hline & & $\mathrm{A} 1 \rightarrow \mathrm{A} 2 \rightarrow \mathrm{A} 3 \rightarrow \mathrm{A} 4 \rightarrow \mathrm{A} 5$ & Vermelho & 2 \\
\hline & & Vermelho & $\mathrm{A} 5 \rightarrow \mathrm{B} 4 \rightarrow \mathrm{A} 3 \rightarrow \mathrm{B} 2 \rightarrow \mathrm{A} 1$ & \\
\hline & & $\mathrm{A} 5 \rightarrow \mathrm{A} 4 \rightarrow \mathrm{A} 3 \rightarrow \mathrm{A} 2 \rightarrow \mathrm{A} 1$ & Verde $-\mathrm{B} 1 \rightarrow \mathrm{A} 2 \rightarrow \mathrm{B} 3 \rightarrow \mathrm{A} 4 \rightarrow \mathrm{B} 5$ & 2 \\
\hline & & $\begin{array}{c}\text { Verde } \\
\mathrm{B} 1 \rightarrow \mathrm{B} 2 \rightarrow \mathrm{B} 3 \rightarrow \mathrm{B} 4 \rightarrow \mathrm{B} 5\end{array}$ & $\begin{array}{l}\text { Vermelho } \\
\text { V51 }\end{array}$ & $?$ \\
\hline & & $\begin{array}{c}\text { Vermelho } \\
\mathrm{B} 5 \rightarrow \mathrm{B} 4 \rightarrow \mathrm{B} 3 \rightarrow \mathrm{B} 2 \rightarrow \mathrm{B} 1\end{array}$ & $\mathrm{DO} \rightarrow \mathrm{A} 4 \rightarrow \mathrm{DO} \rightarrow \mathrm{AL} \rightarrow \mathrm{DI}$ & 2 \\
\hline
\end{tabular}




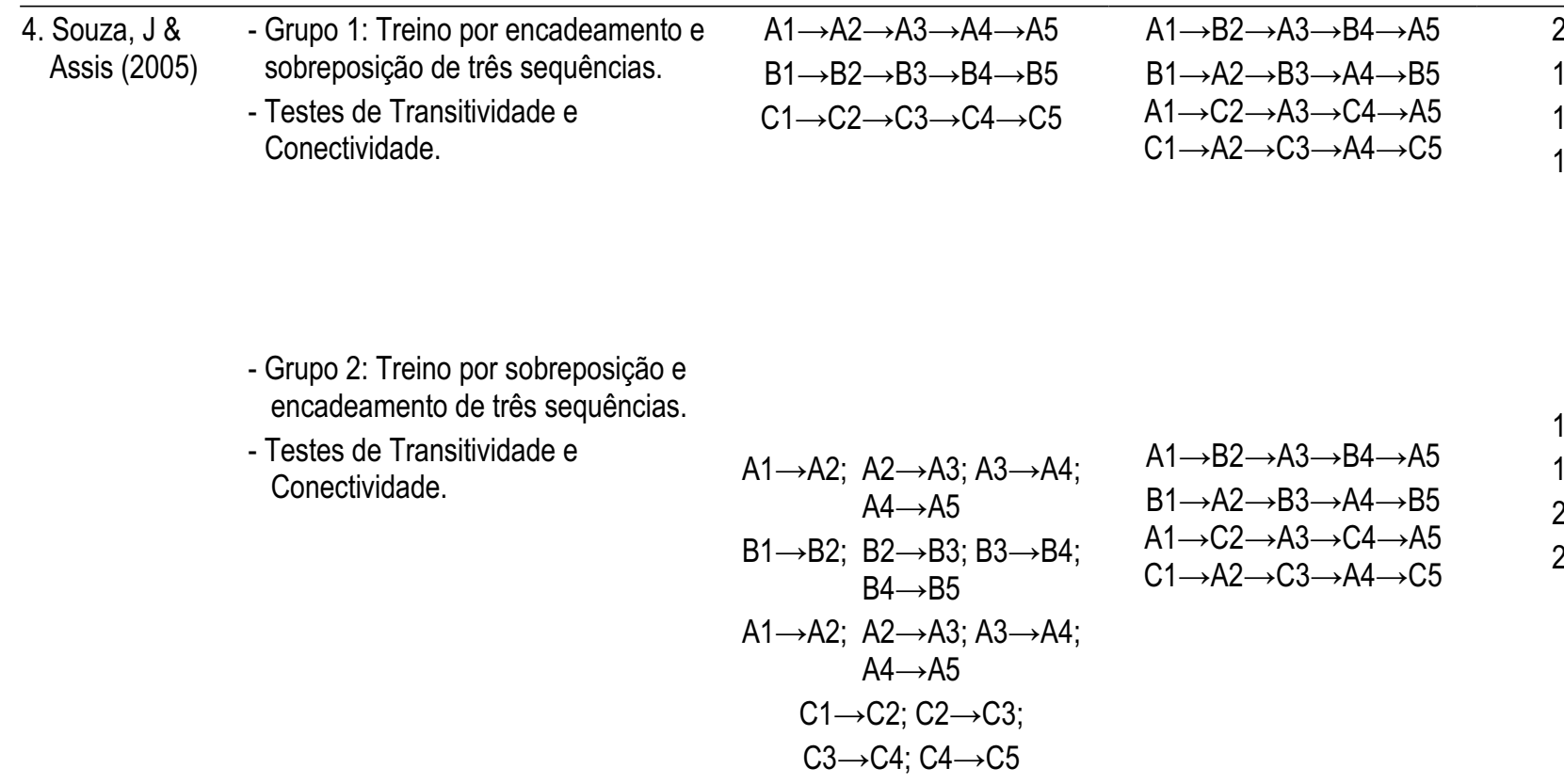

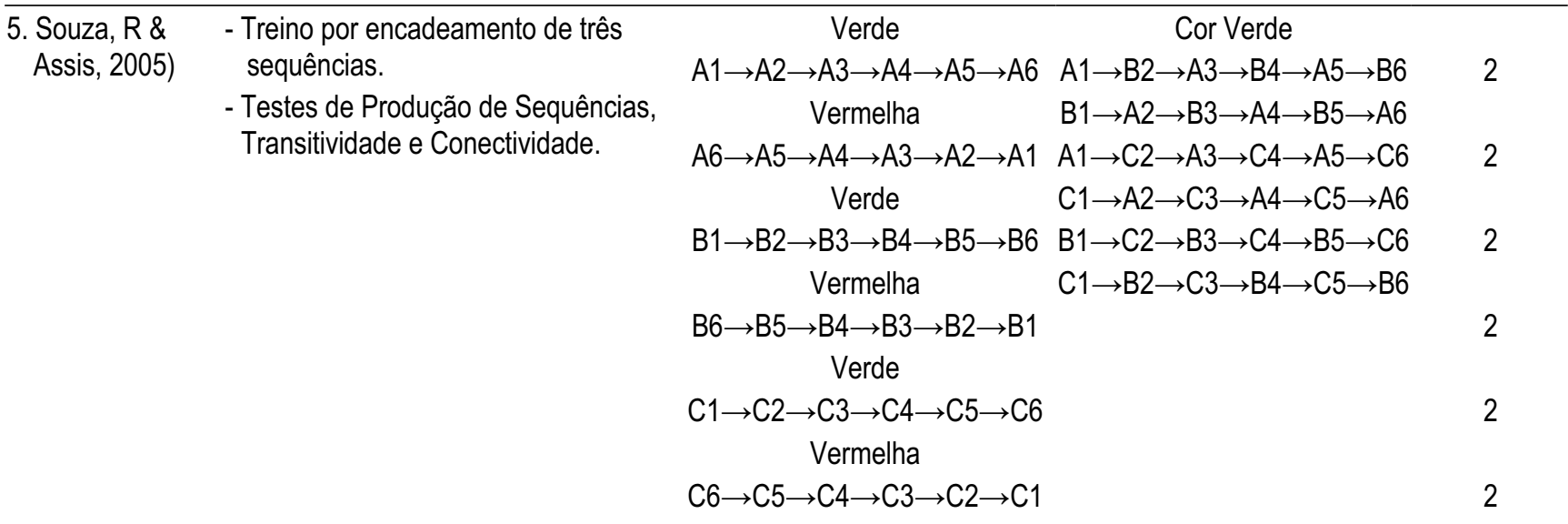




\begin{tabular}{|c|c|c|c|c|}
\hline \multirow{8}{*}{$\begin{array}{l}\text { 6. Sampaio \& } \\
\text { Assis (2005) }\end{array}$} & - Condição 1 & $\mathrm{X} 1 \rightarrow \mathrm{X} 2 \rightarrow \mathrm{X} 3 \rightarrow \mathrm{X} 4 \rightarrow \mathrm{X} 5$ & $\mathrm{X} 1 \rightarrow \mathrm{Y} 2 \rightarrow \mathrm{X} 3 \rightarrow \mathrm{Y} 4 \rightarrow \mathrm{X} 5$ & \multirow{3}{*}{1} \\
\hline & \multirow{2}{*}{$\begin{array}{l}\text { - Treino por Encadeamento de quatro } \\
\text { sequências. }\end{array}$} & $\mathrm{Y} 1 \rightarrow \mathrm{Y} 2 \rightarrow \mathrm{Y} 3 \rightarrow \mathrm{Y} 4 \rightarrow \mathrm{Y} 5$ & $\mathrm{Y} 1 \rightarrow \mathrm{X} 2 \rightarrow \mathrm{Y} 3 \rightarrow \mathrm{X} 4 \rightarrow \mathrm{Y} 5$ & \\
\hline & & $\mathrm{W} 1 \rightarrow \mathrm{W} 2 \rightarrow \mathrm{W} 3 \rightarrow \mathrm{W} 4 \rightarrow \mathrm{W} 5$ & $\mathrm{~W} 1 \rightarrow \mathrm{Z} 2 \rightarrow \mathrm{W} 3 \rightarrow \mathrm{Z} 4 \rightarrow \mathrm{W} 5$ & \\
\hline & $\begin{array}{l}\text { - Testes de Produção de Sequências, } \\
\text { Transitividade e Conectividade. }\end{array}$ & $\mathrm{Z} 1 \rightarrow \mathrm{Z} 2 \rightarrow \mathrm{Z} 3 \rightarrow \mathrm{Z} 4 \rightarrow \mathrm{Z} 5$ & $\mathrm{Z} 1 \rightarrow \mathrm{W} 2 \rightarrow \mathrm{Z} 3 \rightarrow \mathrm{W} 4 \rightarrow \mathrm{Z} 5$ & 1 \\
\hline & & & & 1 \\
\hline & - Condição 2 & $\mathrm{~A} 1 \rightarrow \mathrm{A} 2 \rightarrow \mathrm{A} 3 \rightarrow \mathrm{A} 4 \rightarrow \mathrm{A} 5$ & $\mathrm{~A} 1 \rightarrow \mathrm{B} 2 \rightarrow \mathrm{A} 3 \rightarrow \mathrm{B} 4 \rightarrow \mathrm{A} 5$ & \\
\hline & $\begin{array}{l}\text { - Treino por encadeamento de duas } \\
\text { sequências. }\end{array}$ & $\mathrm{B} 1 \rightarrow \mathrm{B} 2 \rightarrow \mathrm{B} 3 \rightarrow \mathrm{B} 4 \rightarrow \mathrm{B} 5$ & $\mathrm{~B} 1 \rightarrow \mathrm{A} 2 \rightarrow \mathrm{B} 3 \rightarrow \mathrm{A} 4 \rightarrow \mathrm{B} 5$ & 1 \\
\hline & $\begin{array}{l}\text { - Testes de Produção de Sequências, } \\
\text { Transitividade e Conectividade }\end{array}$ & & & \\
\hline
\end{tabular}

7. Nunes \& Assis - Treino AB, AC. Testes de Simetria

(2006)

$\mathrm{A} 1 \rightarrow \mathrm{A} 2 \rightarrow \mathrm{A} 3 / \mathrm{A} 4 \quad \mathrm{~A} 1 \rightarrow \mathrm{B} 2 \rightarrow \mathrm{C} 3 / \mathrm{C} 4$
$\mathrm{~B} 1 \rightarrow \mathrm{C} 2 \rightarrow \mathrm{A} 3 / \mathrm{A} 4$
$\mathrm{C} 1 \rightarrow \mathrm{A} 2 \rightarrow \mathrm{B} 3 / \mathrm{B} 4$
$\mathrm{~B} 1 \rightarrow \mathrm{A} 2 \rightarrow \mathrm{C} 3 / \mathrm{C} 4$
$\mathrm{~A} 1 \rightarrow \mathrm{C} 2 \rightarrow \mathrm{B} 3 / \mathrm{B} 4$
$\mathrm{C} 1 \rightarrow \mathrm{B} 2 \rightarrow \mathrm{A} 3 / \mathrm{A} 4$

Nota: 0 número "1" significa que os participantes responderam prontamente ao teste (primeira tentativa). 0 número "2" indica que os participantes responderam na segunda tentativa (com atraso).

\section{OBJETIVOS DOS ESTUDOS, RESULTADOS E DISCUSSÃO DOS AVANÇOS PRODUZIDOS}

O objetivo do Estudo 1 (Lima \& Assis, 2003) foi verificar se novas sequências seriam produzidas após um formato de treino por pareamento consistente de estímulos, $\mathrm{AB}, \mathrm{AC}, \mathrm{AD}$, sem consequências diferenciais (ver Baptista \& Assis, 1995), seguido de testes de simetria correspondentes. Os estímulos do conjunto "A" foram numerais e os estímulos dos demais conjuntos formas abstratas. O responder sequencial ocorreu na presença dos estímulos "B", "C" e "D". Por exemplo, nos testes de transitividade, as relações emergentes foram: $\mathrm{B} 1 \rightarrow \mathrm{B} 3$; $\mathrm{B} 1 \rightarrow \mathrm{B} 4 ; \mathrm{B} 1 \rightarrow \mathrm{B} 5 ; \mathrm{B} 2 \rightarrow \mathrm{B} 4$; $\mathrm{B} 2 \rightarrow \mathrm{B} 5 ; \quad \mathrm{B} 3 \rightarrow \mathrm{B} 5 ; \quad \mathrm{C} 1 \rightarrow \mathrm{C} 3 ; \quad \mathrm{C} 1 \rightarrow \mathrm{C} 4 ; \quad \mathrm{C} 1 \rightarrow \mathrm{C} 5$; $\mathrm{C} 2 \rightarrow \mathrm{C} 4 ; \quad \mathrm{C} 2 \rightarrow \mathrm{C} 5 ; \quad \mathrm{C} 3 \rightarrow \mathrm{C} 5 ; \quad \mathrm{D} 1 \rightarrow \mathrm{D} 3 ; \quad \mathrm{D} 1 \rightarrow \mathrm{D} 4$; $\mathrm{D} 1 \rightarrow \mathrm{D} 5 ; \mathrm{D} 2 \rightarrow \mathrm{D} 4$; D2 $\rightarrow$ D5; D3 $\rightarrow$ D5; Nos testes de múltipla substituição de estímulos (conectividade) foram: $\mathrm{C} 1 \rightarrow \mathrm{B} 2 \rightarrow \mathrm{C} 3 \rightarrow \mathrm{B} 4 \rightarrow \mathrm{B} 5 ; \mathrm{B} 1 \rightarrow \mathrm{C} 2 \rightarrow \mathrm{C} 3 \rightarrow \mathrm{B} 4 \rightarrow \mathrm{C} 5$; $\mathrm{D} 1 \rightarrow \mathrm{B} 2 \rightarrow \mathrm{D} 3 \rightarrow \mathrm{B} 4 \rightarrow \mathrm{D} 5 ; \mathrm{B} 1 \rightarrow \mathrm{D} 2 \rightarrow \mathrm{B} 3 \rightarrow \mathrm{D} 4 \rightarrow \mathrm{B} 5$.
Os participantes MOI, ATI e LUC precisaram ser expostos a blocos de 20 tentativas nos testes de simetria BA, CA e DA (não apresentados na tabela). Esses resultados indicam que houve uma exposição similar às tentativas de linha de base.

Considerando esses resultados e o procedimento, especificamente quanto à natureza de alguns dos conjuntos de estímulos utilizados (numerais), o Estudo 1 replica, ainda que parcialmente, o estudo de Baptista e Assis (1995). A replicação é parcial, pelo fato de que, no Estudo 1, os demais testes que documentam a equivalência de estímulos não foram aplicados. No teste de Conectividade (envolvendo estímulos dos conjuntos B, C e D), os participantes MOI e ATI precisaram de duas exposições para formar corretamente cada uma das quatro sequências. O participante LUC precisou de uma única exposição para formar corretamente as duas primeiras sequências e de duas exposições para formar corretamente as duas 
últimas. Esses últimos resultados indicam que o Estudo 1 pode ser inserido numa série dos que foram realizados com o propósito de ampliar o estudo de Baptista e Assis (1995), em termos da busca de outras condições em que fosse possível obter desempenhos emergentes a partir de formatos de treino por pareamento consistente de estímulos, sem consequências diferenciais imediatas (Assis, Baptista, Damin \& Álvares, 1997; Assis, Baptista \& Menezes, 2006). Nesse sentido, o estudo produziu avanço em relação aos mencionados, pois foi o primeiro em que um formato de treino por pareamento consistente de estímulos (ver Tabela 1) serviu como linha de base para a avaliação posterior da emergência de novas sequências, por meio de um dos testes que a documentam, o de conectividade.

O objetivo do Estudo 2 (Assis \& Sampaio, 2003) foi explicitar os efeitos da ordem de treino por sobreposição e encadeamento sobre a formação de sequências, demonstrada por meio de testes apropriados. Os resultados dos dois grupos (RUG, HSN, RLS, do Grupo 1, e EFS, CEL, ETS, do Grupo 2), foram idênticos, mostrando que, tanto nos treinos que começaram pela sobreposição como nos que iniciaram pelo encadeamento, os participantes precisaram ser expostos a três tentativas para formar corretamente as quatro sequências envolvendo os conjuntos de estímulos $\mathrm{X}$ e W. Assim, os dois tipos de treinos não diferiram um do outro, em termos de ordem. Porém, o treino por encadeamento foi mais eficiente do que o treino por sobreposição na geração de novas sequências demonstradas no teste de conectividade, em termos de número de tentativas de teste.

O Estudo 2 produziu um avanço em relação ao primeiro. Comparado ao desempenho dos participantes do Estudo 1, na formação de novas sequências avaliadas no teste de Conectividade, o desempenho dos participantes do Estudo 2, segundo grupo, pode ser visto como resultado de um treino mais efetivo de linha de base; ou seja, no Estudo 2, a formação de sequências (inicias) foi estabelecida na linha de base por Encadeamento, diretamente, enquanto que no Estudo 1, o treino de linha de base não envolveu o estabelecimento explícito de sequências, mas sim, de discriminações condicionais entre estímulos sendo que um conjunto deles continha membros (numerais cardinais) que se referiam, cada um, a quantidades diferentes e estas poderiam estar na base da formação de sequência, implicitamente, que passou a gerar outras novas, no teste. É provável que o formato de treino dessas discriminações condicionais tenha dificulta- do o desempenho no teste, relativamente ao formato do treino de linha de base, por Encadeamento, não obstante a diferença do tipo de participantes; isto é, no Estudo 1, com adultos, presume-se que a formação de sequências já fazia parte do repertório comportamental.

No Estudo 3, com dois experimentos, conduzido com crianças com desenvolvimento típico, pretendeu-se no primeiro experimento, avaliar a emergência de classes ordinais, após treino com quatro conjuntos de estímulos diferentes, por encadeamento. Os testes para a produção de novas sequências foram os mesmos do Estudo 2 (transitividade e conectividade). Os resultados mostraram que os participantes responderam consistentemente com a linha de base adotada, alguns na segunda tentativa. Objetivou-se, no Experimento 2, estender os resultados do experimento anterior, para avaliar se o responder ordinal ainda se manteria estável quando os participantes respondessem sob controle condicional e se os estímulos envolvidos seriam funcionalmente equivalentes. Pode-se considerar, no Estudo 3, que os resultados nos testes de conectividade aplicados aos participantes após o treino por Encadeamento simples (Experimento 1) foram similares aos dos participantes do Estudo 2 (Grupo 2) cujo desempenho na formação de sequência foi também iniciada por encadeamento simples.

No Estudo 4 (Souza, J \& Assis, 2005), buscou-se explicitar os efeitos do ensino por encadeamento e por sobreposição em duas condições experimentais com dois conjuntos diferentes de estímulos, sobre o responder sequencial. Os resultados do Estudo 4, comparativamente aos do Estudo 3 (Experimento 1) nos testes de conectividade, indicam que houve um avanço daquele em relação a este, pois o encadeamento confirmou-se efetivo como procedimento de linha de base para a formação de novas sequências, replicando, no Estudo 4, os resultados com outros tipos de participantes (alunos com atraso no desenvolvimento cognitivo, ou seja, com retardo mental de leve a moderado). Dos seis participantes do Estudo 4, cinco precisaram de apenas uma exposição ao teste de conectividade, resultados esses que foram similares aos do estudo anterior, em termos de número de exposição. Pode-se considerar que os resultados do Estudo 4 replicaram os obtidos no Estudo 3 (Experimento 2) que foi conduzido com crianças com desenvolvimento típico.

O objetivo do Estudo 5 (Souza \& Assis, 2005) foi avaliar, em alunos com surdez congênita, a emergência do controle condicional exercido por duas cores diferentes (verde e vermelha) sobre a formação de 
sequências envolvendo sinais em LIBRAS (Língua Brasileira de Sinais) e avaliar se os estímulos de cada sequência seriam funcionalmente equivalentes. Mesmo considerando a complexidade do procedimento do Estudo 4, aponta-se que a manipulação do controle condicional adicionado ao encadeamento, no estudo, mostrou-se efetiva em participantes surdos, o que constituiu um avanço em relação ao Estudo 4.

Foi objetivo do Estudo 6 (Sampaio \& Assis, 2005) ampliar os resultados obtidos por Assis e Sampaio (2003) para outros participantes (alunos com atraso no desenvolvimento cognitivo), avaliando os efeitos de uma história de treino por encadeamento de duas sequências em condições diferentes quanto aos estímulos experimentais (usuais e não usuais), sobre o desempenho em testes e verificar se após a emergência de classes sequenciais os estímulos envolvidos seriam funcionalmente equivalentes. Os resultados não indicaram ter havido um avanço em relação aos do Estudo 4 , realizado com os mesmos tipos de participantes, pois no Estudo 6, dos três participantes, um não demonstrou a formação de nenhuma sequência nova, nos testes de conectividade e outro foi bem-sucedido nesses testes, apenas parcialmente. É provável que essa não replicação seja explicada pelo fato de, no Estudo 6 , o ensino por encadeamento ter sido arbitrário, envolvendo estímulos diferentes entre si, ao passo que, no Estudo 4, o ensino por encadeamento envolveu estímulos idênticos quanto à forma, mas diferentes quanto ao tamanho - a dimensão crítica nesse ensino.

O objetivo de Estudo 7 (Nunes \& Assis, 2006) foi verificar se classes ordinais poderiam emergir no teste de conectividade, após o ensino de relações condicionais entre estímulos de três conjuntos, seguido do estabelecimento de um encadeamento com estímulos de um desses conjuntos. Os resultados indicam que os desempenhos dos participantes, relativamente aos desempenhos dos participantes do Estudo 6, não apresentaram um avanço, se for considerada a pequena diferença entre os desempenhos nos dois estudos; essa diferença pode ser vista como compensada pela diferença nos tipos de participantes dos dois estudos (alunos com atraso no desenvolvimento cognitivo, no Estudo 7, e adultos, no Estudo 1) e pela não correspondência precisa entre os procedimentos utilizados nos estudos (ver Tabela 1). Em termos gerais, entretanto, os resultados do Estudo 7 sugerem a efetividade dos aspectos de procedimento que foram comuns, ou seja, a aplicação de testes de conectividade após o ensino de relações condicionais.

\section{CONSIDERAÇÕES FINAIS E QUESTÕES PARA FUTURAS PESQUISAS}

Os estudos relatados aqui apresentam em comum o fato do treino por encadeamento ou sobreposição de estímulos constituir a linha de base para exposição aos testes de transitividade, conectividade e formação de classes ordinais. O que caracteriza uma cadeia operante é que as respostas são ensinadas em sequência, de modo que para cada uma das respostas haja um estímulo com dupla função - ser reforçador para a resposta anterior e discriminativo para a seguinte. Assim, o treino por encadeamento é um procedimento que diminui a probabilidade de erros. O desempenho, com regularidade, demonstrado pelos participantes dos estudos referidos no presente trabalho atesta a viabilidade desse tipo de treino. Mesmo nos estudos de Assis e Sampaio (2003) e de Souza e Assis (2005), os grupos de participantes que foram submetidos tanto ao treino por encadeamento quanto ao treino por sobreposição apresentaram um desempenho melhor, como resultado do primeiro procedimento, independentemente da natureza dos estímulos experimentais.

Tradicionalmente, o treino por encadeamento tem sido utilizado como linha de base para o teste posterior de formação de sequências, por exemplo, o teste de conectividade. Nos estudos apontados neste trabalho, em geral, os resultados nesse teste foram regulares, no sentido de que os participantes responderam de imediato ou com atraso (Green \& Saunders, 1998), isto é, após uma segunda exposição às tentativas. Pode-se questionar, entretanto, o caráter rigorosamente emergente das relações demonstradas nesse teste, se for levado em conta que ocorreram depois do estabelecimento de uma de linha de base cujo formato foi de encadeamento. Em algum momento desse treino, os estímulos experimentais foram apresentados conjuntamente (por exemplo, A1 $\rightarrow \mathrm{A} 2 \rightarrow \mathrm{A} 3 \rightarrow \mathrm{A} 4 \rightarrow \mathrm{A} 5$ ) ao participante. Assim, esse formato de treino pode ser visto como indutor do desempenho demonstrado no teste.

No treino por sobreposição, o primeiro estímulo é removido, o segundo estímulo passa a exercer a função ordinal de primeiro e um terceiro estímulo introduzido na sequência programada exerce a função ordinal de segundo. Portanto, essa reversão de funções ordinais pode dificultar o responder sequencial com esse procedimento (Lopes Junior \& Agostini, 2004).

Têm sido de modalidade visual os estímulos experimentais dos estudos relacionados ao desempenho sequencial. Um pareamento auditivo-visual de estí- 
mulos é um procedimento relevante a ser considerado no treino, o que não foi contemplado nos estudos sobre produção de sequência. Um estudo conduzido por Green (1990) indicou o efeito mais acurado de estímulos visuais e auditivos sobre discriminações condicionais, não obstante o procedimento ter sido de pareamento com o modelo.

Na literatura, bem como nos estudos mencionados neste trabalho, tem-se constatado o uso de sequências de no máximo seis estímulos, com participantes humanos. Uma ampliação do número de estímulos membros da classe e, consequentemente, da recombinação dos mesmos, no treino, poderá ser uma variável a analisar. Essa recombinação poderá ampliar a amostra de estímulos a comporem os testes de relações emergentes.

Nos estudos mencionados neste trabalho, os estímulos experimentais foram formas abstratas, exceto aqueles dos estudos de Assis e Sampaio (2003). Atribui-se à natureza dos estímulos desse estudo, ou seja, o fato de terem sido figuras usuais, o papel facilitador do responder dos participantes. Outros estudos poderão continuar a investigar o efeito desta variável.

Pode-se conceber a variável ordem de apresentação dos estímulos como relevante à formação de sequência; em outras palavras, como uma fonte de controle do responder sequencial. É nesse sentido, conforme se apontou anteriormente, que a análise de Green e cols. (1993) é retomada por Carmo (2002), não obstante a divergência considerada. Se o exemplo de Carmo (2002) - referente à criança que, ao sequenciar brinquedos, o faz sob controle do tipo de material de que são confeccionados - não elucida o sequenciar como sendo ordenar, então uma questão se coloca: com base em quê a criança escolhe primeiramente os brinquedos de plástico e, em seguida, os de madeira? Uma resposta admissível é que não seja identificável, de imediato, a base desse desempenho, o que não configura, entretanto, inexistência de fonte de controle outra que não seja o tipo de material. Resposta alternativa pode ser buscada numa possível história de relação da criança com brinquedos de determinado material; relação essa em que o tipo de material deva ter determinado a ordem de escolha do brinquedo. Assim, hipoteticamente, o tipo de material remete a essa história de relação, revelando o controle do desempenho sequencial. Se a hipótese se aplica, então a determinação da ordem de escolha dos brinquedos por tipo de material é pertinente e, em decorrência disso, dispor brinquedos em sequência é, em última instância, ordenar.
Essa análise permite considerar que, no responder sequencial, a variável ordem tem sentido ou se define a partir de dimensões ou características de estímulo com as quais tem relação. A numerosidade, o tamanho, a forma, a substância e outras são algumas dessas dimensões ou características. Por exemplo, pode-se escolher (responder a) primeiramente objetos grandes (ou redondos, ou de vidro, ou que têm neles acoplado um número grande de figuras); em segundo lugar, objetos pequenos (ou quadrados, ou de cerâmica, ou que têm neles acoplado um número pequeno de figuras); em terceiro lugar, objetos ainda menores (ou triangulares, ou de madeira, ou que têm neles acoplado um número menor ainda de figuras) e assim por diante. Cada uma dessas escolhas indica o controle pela ordem: responder primeiramente a objetos conforme o maior tamanho, ou forma arredondada, ou com maior número de figuras e, por último, responder a objetos de menor tamanho, ou de formato triangular, ou de madeira, ou com menor número de figuras. Há que buscar o êxito das escolhas na história de treino discriminativo da ordem (decrescente, que, no caso, revela a formação dos conceitos maior e menor), de treino discriminativo das dimensões relevantes dos estímulos - numerosidade, forma, substância - e ainda da relação (sobreposição) entre cada uma dessas dimensões e a ordem (decrescente) pela qual são escolhidos os estímulos de cada vez. Por exemplo, a numerosidade como propriedade discriminativa relevante, pode levar à emergência de novas sequências sem qualquer treino adicional. A descrição de topografias de controle de estímulos (TCE) apresentada na introdução deste trabalho poderia ser ampliada, uma vez que esse termo não se restringe apenas a propriedades físicas dos estímulos, mas a propriedades relacionais também. Nos estudos que investigam o papel das TCEs durante o estabelecimento de relações condicionais e suas implicações para a emergências de relações de equivalência, além de propriedades físicas são destacadas as propriedades relacionais (p. ex. controle por $\mathrm{S}+$, por $\mathrm{S}-$ ou por ambos os estímulos e, cada relação de controle tem implicações na formação de classes). Embora com necessidade de mais investigações, a variável ordem poderia ou parece ser a TCE relevante nesse caso.

Estudos como o de Souza, Assis e Magalhães (2005) indicam que o efeito do treino de uma sequência com estímulos envolvendo a numerosidade como propriedade discriminativa relevante, precisa ser analisado; isso poderá ser feito, por exemplo, em um estudo que compare a aquisição de comportamento ordinal emer- 
gente, após o estabelecimento de treinos em que haja estímulos com e sem essa propriedade. A sobreposição desta propriedade (numerosidade) à contingência de discriminação simples baseada no responder sequencial torna ainda mais clara a possibilidade de se estudar a aquisição de comportamentos conceituais numéricos no contexto dos estudos sobre comportamento ordinal emergente, conforme a proposta de Green e cols. (1993).

$\mathrm{Na}$ literatura, bem como nos estudos mencionados neste trabalho, não têm sido aplicados testes de generalização nem verificado a estabilidade do desempenho pós-experimental, decorrido algum tempo (Spradlin, Saunders \& Saunders, 1992). Essa verificação é um importante procedimento para averiguar a efetividade do treino e dos testes, para além do período de duração do experimento.

Nos estudos mencionados neste trabalho, a topografia de resposta foi apontar ou tocar na tela do monitor. Outras formas que possibilitem monitorar respostas encobertas, como as verbais envolvidas na formação de classes sintáticas, ao longo do experimento, além de respostas motoras, poderão ser investigadas. Monitorar respostas encobertas é um desafio, devido à dificuldade que acarreta; porém, poderá ser de grande valia na avaliação do seu papel na formação de sequências.

Respostas musicais constituem um tipo de respostas verbais interessantes de serem consideradas. Uma das razões para esse interesse é que, diferentemente do desempenho sequencial habitual (rotinas), as respostas musicais não se caracterizam necessariamente como formação de sequências apenas ascendentes (crescentes) ou apenas descendentes (decrescentes), mas há casos em que constituem esses dois tipos combinados, sem uma linearidade. Um determinado trecho musical (melodia) pode ter as notas dó, ré, mi, fá, sol (sequência ascendente), ou as notas sol, fá, mi, ré, dó (sequência descendente), posicionadas na partitura sob o indicativo de uma mesma clave, havendo entre cada nota apenas um tom ou semitom de diferença. Outro trecho pode ter as notas dó, ré, mi, fá, dó, ré, dó, em que as quatro primeiras notas formam uma sequência ascendente, sendo a quinta o retorno à nota inicial (descendente), seguida de uma nota ascendente, e da última como retorno (descendente) à primeira.

Os estudos em que houve o treino por encadeamento, como também aqueles em que houve o treino por sobreposição, as sequências envolvidas têm sido sequências repetitivas e simples. Poderá ser investiga- da a utilização de sequências não repetitivas e complexas. Levantar-se, realizar tarefas de higiene pessoal, tomar o café da manhã e dirigir-se ao local de trabalho podem ser atividades fracionadas de modo a constituírem componentes detalhados de sequências repetitivas ou estereotipadas. Tanto é verdade que qualquer interrupção da cadeia impede a sua integridade e, com isso, o reforçamento. Há situações em que é necessário alterar respostas rotineiras, em virtude de sua repetição ser altamente aversiva (cf. Brady, Saunders \& Spradlin, 1994). Importantes autoridades mudam a rotina pela qual realizam tarefas, como deslocar-se da residência ao trabalho e/ou vice-versa, por ser esse um meio de evitar sequestros, assaltos ou outros atentados à sua integridade.

Sequências simples podem ser caracterizadas como aquelas em que as respostas são operantes de um mesmo tipo, como as respostas motoras de tocar ou apontar para um estímulo determinado. Sequências de respostas verbais ou de respostas motoras e verbais em conjunto, sequências não lineares, podem ser consideradas como complexas.

A variável ordem relaciona-se a dimensões de estímulo, dentre as quais o tamanho e a temporalidade caso específico que remete à relação entre eventos antecedentes e eventos consequentes. A aprendizagem da classificação dos filhos pela ordem de nascimento é um exemplo de desempenho sequencial em que o primeiro (filho) necessariamente é apontado com base no evento que ocorreu antes, relativamente aos eventos que o sucederam. Aprender a discriminar o ontem relativamente ao hoje e ao amanhã, bem como classificar pessoas segundo o papel social, político ou econômico (função na hierarquia) que desempenham, por exemplo, numa instituição, seguem o mesmo padrão. É importante investigar outros aspectos relacionados ao status da ordem na formação de sequências. Outro exemplo é a formação de classes sintáticas em que a disposição das palavras em uma frase segue uma determinada ordem, estabelecida pela comunidade verbal para poder ter sentido; essa ordem pode mudar em função da língua: português, inglês, mandarim.

Este último aspecto e os demais já referidos poderão constituir questões para futuras pesquisas. Os resultados a serem obtidos poderão produzir avanços teórico-metodológicos no que diz respeito à investigação de variáveis de controle do responder sequencial, além daquelas envolvidas nos estudos considerados no presente trabalho. 


\section{REFERÊNCIAS}

Assis, G. J. A., \& Costa, L. C. A. (2004). Emergência de relações ordinais em crianças. Interação em Psicologia, 8(2), 199-216.

Assis, G. J. A., \& Sampaio, M. E. C. (2003). Efeitos de dois procedimentos de ensino para formação de classes sequenciais. Interação em Psicologia, 7(2), 52-63.

Assis, G. J. A., Baptista, M. Q. G., \& Menezes, A. B. (2006). Discriminações condicionais após treino de pareamento consistente de estímulos complexos com atraso. Estudos de Psicologia, 11(2), 217-228.

Assis, G. J. A., Baptista, M. Q. G., Damin, E. T. B., \& Álvares, S. M. M. (1997). Consistency training and equivalence relations without differential consequences. Experimental Analysis of Human Behavior Bulletin, 15(1), 6-7.

Baptista, M. Q. G., \& Assis, G. J. A. (1995). Treino por consistência de estímulos sem consequências diferenciais. Psicologia: Teoria e Pesquisa, 11(3), 173-179.

Binder, C. (1996). Behavioral fluency: Evolution of a new paradigm. The Behavior Analyst, 19, 163-197.

Brady, N. C., Saunders, K. J., \& Spradlin, J. E. (1994). A conceptual analysis of request teaching procedures for individuals with severely limited verbal repertoires. The Analysis of Verbal Behavior, 12, 43-52.

Carmo, J. S. (2002). Definições operacionais de habilidades matemáticas elementares. Em H. J. Guilhardi, M. B. B. P. Madi, P. P. Queiroz \& M. C. Scoz (Orgs.), Sobre comportamento e cognição: Vol. 9. Contribuições para a construção da teoria do comportamento (pp. 181-191). Santo André: ESETec.

Catania, A. C. (1999). Aprendizagem: Comportamento, linguagem e cognição (D. Souza, Trad.) ( $4^{\mathrm{a}}$ ed.). Porto Alegre: Artes Médicas.

Dube, W., \& Macllvane, W. (1996). Implications of a stimulus control topography analysis for emergent behavior and stimulus classes. Em T. R. Zentall \& P. M. Smeets (Orgs.), Stimulus class formation in humans and animals (pp. 197-218). Amsterdam: Elsevier.

Galy, E., Camps, J. F., \& Melan, C. (2003). Sequence class formation following learning of short sequences. The Psychological Record, 53, 635-645.

Green, G. (1990). Differences in development of visual and auditory-visual equivalence relations. American Journal on Mental Retardation, 95(3), 260-270

Green, G., \& Saunders, R. R. (1998). Stimulus equivalence. Em K. Lattal \& M. Perone (Orgs.), Handbook of research methods in human operant behavior (pp. 229-262). New York: Plenum Press.

Green, G., Sigurdardottir, Z. G., \& Saunders, R. R. (1991). The role of instructions in transfer of ordinal functions through equivalence classes. Journal of the Experimental Analysis of Behavior, 55, 287-304.

Green, G., Stromer, R., \& Mackay, H. (1993). Relational learning in stimulus sequences. The Psychological Record, 43, 599-616.

Holcomb, W. L., Stromer, R., \& Mackay, H.A (1997). Transitivity and emergent sequence performance in young children. Journal of Experimental Child Psychology, 65, 96-124.
Johnson, K. R., \& Layng, T. V. J. (1996). On terms and procedures: Fluency. The Behavior Analyst, 19, 281-288.

Kelleher, R. T. (1996). Chaining and conditioned reinforcement. Em W. K. Honig (Org.), Operant behavior: Areas of research and application (pp. 160-212). New York: Appleton-CenturyCrofts.

Lazar, R. (1977). Extending sequence-class membership with matching to sample. Journal of the Experimental Analysis of Behavior, 27, 381-392.

Lazar, R., \& Kotlarchyk, B. J. (1986). Second order control of sequence class equivalence in children. Behavioural Processes, 13, 205-215.

Lima, M. P., \& Assis, G. J. A. (2003). Emergência de classes sequenciais após treino com pareamento consistente. Psicologia: Teoria e Pesquisa, 19, 75-84.

Lopes Junior, J., \& Agostini, M. F. (2004). Aquisição e emergência de relações ordinais por crianças da segunda série do ensino fundamental. Em O. M. P. R. Rodrigues, T. G. M. Valle, A. C. M. Verdu, J. Lopes Júnior \& M. R. Cavalcante (Orgs.), Psicologia do desenvolvimento e aprendizagem: Investigações e análises (pp. 95-127). São Carlos: Rima.

Mackay, H. A., Kotlarchyk, B. J., \& Stromer, R. (1997). Stimulus classes, stimulus sequences, and generative behavior. Em D. M. Baer \& E. M. Pinkston (Orgs.), Environment and behavior (pp. 124-137). Boulder: Westview Press.

Maydak, M., Stromer, R., Mackay, H., \& Stoddard, L. (1995). Stimulus classes in matching to sample and sequence production: The emergence of numeric relations. Research in Developmental Disabilities, 16, 179-204.

McIlvane, W. J., \& Dube, W. V. (1992). Stimulus control shaping and stimulus control topographies. The Behavior Analyst, 15, 89-94.

McIlvane, W. J., \& Dube, W. V. (2003). Stimulus control topography coherence theory: Foundations and extensions. The Behavior Analyst, 26, 195-213

Millenson, J. R. (1975). Princípios de análise do comportamento (A. A. Souza \& D. Rezende, Trads.). Brasília: Coordenada. (Original publicado em 1967)

Nunes, A. L. M., \& Assis, G. J. A. (2006). Emergência de classes ordinais após o ensino de relações numéricas. Revista Brasileira de Análise do Comportamento, 2(2), 209-219.

Ribeiro, M. P. L., Assis, G. J. A., \& Enumo, S. R. F. (2005). Controle do comportamento por relações ordinais: Questões conceituais e metodológicas. Em E. B. Borloti, S. R. F. Enumo \& M. P. L. Ribeiro (Orgs.), Análise do comportamento: Teorias e práticas (pp. 117-132). Santo André: ESETec.

Sampaio, M. E. C., \& Assis, G. J. A. (2005). Equivalência de estímulos sequenciais em portadores de necessidades educacionais especiais. Acta Comportamentalia, 13(2), 111-143.

Serna, R. (2004). Recent advance in discrimination learning with individuals with developmental disabilities. Em W. L. Williams (Org.), Developmental disabilities: Etiology, assessment, intervention, and integration. Reno: Context Press.

Sidman, M., \& Tailby, W. (1982). Conditional discrimination vs. matching to sample: An expansion of the testing paradigm. Journal of the Experimental Analysis of Behavior, 37, 5-22. 
Sigurdardottir, Z. G., Green, G., \& Saunders, R. R. (1990). Equivalence classes generated by sequence training. Journal of the Experimental Analysis of Behavior, 53, 47-63.

Skinner, B. F. (1938). The behavior of organisms. New York: Appleton-Century-Crofts.

Skinner, B. F. (2000). Ciência e comportamento humano (J. C. Todorov \& R. Azzi, Trads.) (10 ed.). São Paulo: Martins Fontes.

Souza, J. A. N., \& Assis, G. J. A. (2005). Efeito de dois procedimentos de ensino sobre o comportamento de ordenar. Psicologia em Estudo, 10(3), 527-536.

Souza, R. D. C., \& Assis, G. J. A. (2005). Emergência de relações numéricas em crianças surdas. Psicologia: Teoria e Pesquisa, 21(3), 297-308.

Souza, R. D. C., Assis, G. J. A., \& Magalhães, P. G. (2005). Equivalência numérica em crianças surdas. Temas em Psicologia, 13(2), 113-127.

Stevens, J. (1951). Mathematics, measurement, and psychophysics. Em S. Stevens (Org.), Handbook of experimental psychology (pp. 1-49). New York: John Wiley and Sons.

Spradlin, J. E. (1999). Rotinas: Implicações para a vida e para o ensino. Temas em Psicologia, 7(3), 223-234.

Spradlin, J. E., Saunders, K. J., \& Saunders, R. R. (1992). The stability of equivalence classes. Em Hayes, S. C., \& Hayes, L. J. (Orgs.), Understanding verbal relations (pp. 29-42). Reno: Context Press.
Stromer, R., \& Mackay, H. A. (1990). A note on the study of transitive relation in stimulus sequence. Experimental Analysis of Human Behavior Bulletin, 8, 2-5.

Stromer, R., \& Mackay, H. A. (1992a). Conditional stimulus control of children's sequence production. Psychological Reports, 70, 903-912.

Stromer, R., \& Mackay, H. A. (1992b). Some effects of presenting novel stimuli on a child's sequence production. Experimental Analysis of Human Behavior Bulletin, 10, 21-25.

Stromer, R., \& Mackay, H. A. (1993). Human sequential behavior: Relations among stimuli, class formation, and derived sequences. The Psychological Record, 43, 107-131.

Stromer, R., Mackay, H. A, Cohen, M., \& Stoddard, L. T. (1993). Sequence learning in individuals with behavioral limitations. Journal of Intellectual Disability Research, 37, 243-261.

Wulfert, E., \& Hayes, S. C. (1988). Transfer a conditional ordering response through conditional equivalence classes. Journal of the Experimental Analysis of Behavior, 50, 125-144.

Recebido: 01/08/2008

Última revisão: 15/07/2009 Aceite final: 13/08/2009

Nota:

${ }^{1}$ Agradecemos as sugestões valiosas do Prof. Dr. Olavo de Faria Galvão e ao Prof. William Lee Martin, PhD à versão original deste artigo.

\section{Sobre os autores:}

Grauben José Alves de Assis: Doutor em Psicologia Experimental pela Universidade de São Paulo. Docente da Faculdade de Psicologia e do Programa de Pós-Graduação em Teoria e Pesquisa do Comportamento, Universidade Federal do Pará.

Bolsista de Produtividade em Pesquisa do CNPq.

Marcelo Quintino Galvão Baptista: Doutor em Educação pela Universidade Federal de São Carlos e Docente da Faculdade de Psicologia e do Programa de Pós-Graduação em Teoria e Pesquisa do Comportamento, Universidade Federal do Pará.

Ana Letícia Moraes Nunes: Doutoranda no Programa de Pós-Graduação em Teoria e Pesquisa do Comportamento, Universidade Federal do Pará.

Endereço para correspondência: Grauben José Alves de Assis - Rua Jerônimo Pimentel, 426/1801 - Bairro do Umarizal - 66055-000 Belém/PA - E-mail: grauben@pesquisador.cnpq.br. 